\title{
Prospective Multiaxial Motion Correction for fMRI
}

\author{
Heidi A. Ward, ${ }^{*}$ Stephen J. Riederer, Roger C. Grimm, Richard L. Ehman, \\ Joel P. Felmlee, and Clifford R. Jack, Jr.
}

\begin{abstract}
Corruption of the image time series due to interimage head motion limits the clinical utility of functional MRI. This paper presents a method for real-time prospective correction of rotation and translation in all six degrees of rigid body motion. By incorporating an orbital navigator (ONAV) echo for each of the sagittal, axial, and coronal planes into the fMRI pulse sequence, rotation and translation can be measured and the spatial orientation of the image acquisition sequence that follows can be corrected prospectively in as little as $160 \mathrm{msec}$. Testing of the method using a computerized motion phantom capable of performing complex multiaxial motion showed subdegree rotational and submillimeter translational accuracy over a range of $\pm 8^{\circ}$ and $\pm 8 \mathrm{~mm}$ of motion. In vivo images demonstrate correction of simultaneous through-plane and in-plane motion and improved detection of fMRI activation in the presence of head motion. Magn Reson Med 43:459-469, 2000. () 2000 Wiley-Liss, Inc.
\end{abstract}

Key words: functional magnetic resonance imaging; navigator echoes; motion correction; real-time MRI; functional MRI artifacts

Clinical implementation of functional MRI (fMRI) studies has been hindered by the high prevalence of interimage head motion in patient studies, relative to studies in normal volunteers (1-5). Physical restraints are only partially effective in reducing head motion (6). Retrospective motion-correction algorithms, defined as those performed after collection of all data for a single fMRI run, provide a possible solution to the problem of head motion $(1,3,7-$ 13), but have fundamental drawbacks. These include reliance on interpolation and registration of image data, which introduce blurring and violate the assumption of uniform statistical variance (14). Also, retrospective motion correction is unable to correct for the disrupted spin history resulting from through-plane motion. Prospective motion correction, defined as correction for subject motion before image acquisition, shares none of these drawbacks and has been demonstrated to improve fMRI results $(15,16)$. However, to date, implementation of this technique has been limited to correction of motion in a single plane.

Given the highly flexible anatomy of the cervical spine and craniocervical junction, most head motions during fMRI exams are not oriented precisely about a specific physical axis. Thus, for accurate correction, motions must be detected and corrected concurrently in all three orthogonal planes. The goal of this project was to expand the method for real-time adaptive correction of rotation and

Department of Radiology, Mayo Clinic, Rochester, Minnesota.

Grant sponsor: National Institutes of Health; Grant numbers: CA73691; CA37993.

${ }^{*}$ Correspondence to: Clifford R. Jack, Jr., M.D., Department of Radiology and MRI Research Laboratory, Mayo Clinic, 200 First Street SW, Rochester, MN 55905.

Received 17 May 1999; revised 5 November 1999; accepted 16 November 1999.

(c) 2000 Wiley-Liss, Inc. translation in a single plane into a method that detects and corrects for rotation and translation in all six rigid body degrees of freedom.

\section{METHODS}

\section{Overview}

Throughout this work, we attempt to draw a clear distinction between the separate processes of image acquisition and the acquisition of orbital navigator (ONAV) echoes (17) which are not reconstructed into images. With the prospective real-time correction technique, each set of multislice images is preceded by ONAV echoes in each of the three orthogonal planes. Immediately after their acquisition, the navigator signals are processed to determine motion in all three degrees of rotational freedom and three degrees of translational freedom. The image acquisition parameters are then immediately altered to compensate for any rotation or through-plane translation. The time from acquisition of the first navigator echo to alteration of the ssEPI acquisition can be less than $160 \mathrm{msec}$. Any motion relative to baseline determined from the ONAV acquisitions at the start of the multislice cycle is used to correct all the imaging sections of that cycle. The technique is illustrated in Fig. 1.

Gradient echo single-shot echo planar imaging (ssEPI) was used for image acquisition throughout this work and multislice axial image acquisitions were used for all experiments. The directional convention adopted defined $x$ as the left-right $(\mathrm{L} / \mathrm{R})$ direction, $y$ as the anterior-posterior (A/P) direction, and $z$ as the superior/inferior (S/I) direction along the bore of the magnet. The coronal ONAV has a circular $k$-space trajectory in the $x-z$ plane, the sagittal ONAV has a circular $k$-space trajectory in the $y-z$ plane, and the axial ONAV has a circular $k$-space trajectory in the $x-y$ plane.

\section{Orbital Navigator Echoes}

Rotation and translation of an object within a given plane can be measured from the MR signal from an acquisition of a circular $k$-space trajectory with fixed $k$-space radius $k_{\rho}$ (17). If $S\left(k_{\rho}, \theta\right)$ is the signal from the ONAV echo, then the signal of an object rotated through an angle $\alpha$ and translated by $\left(x_{o}, y_{o}\right)$ can be described as

$$
S^{\prime}\left(k_{\rho}, \theta\right)=S\left(k_{\rho}, \theta-\alpha\right) e^{i k_{\rho}\left(x_{0} \cos \theta+y_{0} \sin \theta\right)} .
$$

Here, $\theta$ is the azimuthal angle in $k$-space at the radius $k_{\mathrm{\rho}}$ of the ONAV echo and is proportional to the time $t$ over which the echo is measured. The ONAV signals from the first application of each of the orthogonal ONAVs are retained as the baseline signals. For subsequent cycles, the signal measured from each ONAV is compared with its 


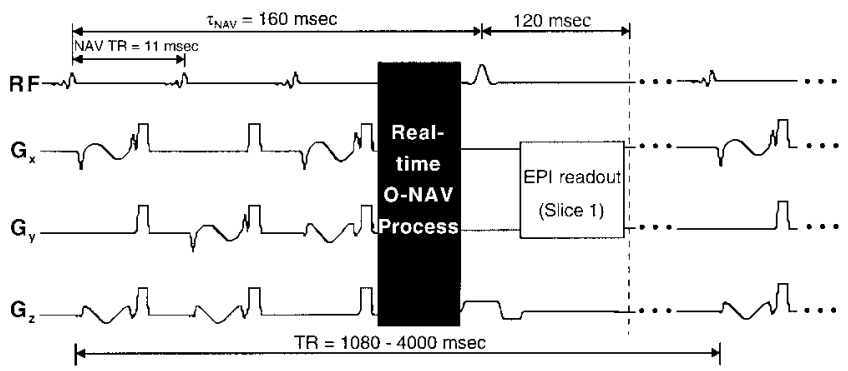

FIG. 1. Pulse sequence diagram. Three ONAV pulse sequences, one for each orthogonal plane, are applied prior to each cycle of multislice ssEPI imaging sequences. During the time indicated in black, the detected angular rotations and through-plane translation are corrected in real time. The entire ONAV process, from RF excitation of the initial ONAV to RF excitation of the corrected EPI sequence, requires $160 \mathrm{msec}$.

respective baseline signal, and hence interimage head motion can be tracked and corrected throughout the fMRI time series acquisition. From Eq. [1], rotation is encoded only in the magnitude of the signal. Thus, measurement of any shift in the magnitude yields the detected rotation. Translation is encoded in the phase of the signal and can be extracted through analysis of the phase.

To correct for the detected rotation, the gradient rotation matrix is rotated about the isocenter of the magnet. When the center of rotation of the object is not coincident with isocenter, rotation of the gradient rotation matrix about isocenter results in an apparent translation of the object (16). Apparent translation is not due to a physical translation of the object with respect to the magnet bore, but rather to translation with respect to its new frame of reference. The sum of the actual and apparent translations is the amount of translation visible in the images, as shown in (16), and must be corrected. If the phase of the reference ONAV signal, $\Psi(\theta)$, is shifted by the detected angular rotation, $\alpha$, then the apparent translation is encoded in the difference of the phase of the reference ONAV signal and the current ONAV signal, $\Psi^{\prime}(\theta)$, along with any actual translation (18)

$$
\begin{aligned}
\Delta \Psi(\theta)=\Psi^{\prime}(\theta)- & \Psi(\theta-\alpha) \\
& =k_{\mathrm{p}}\left[X_{o} \cos (\theta-\alpha)+y_{o} \sin (\theta-\alpha)\right] .
\end{aligned}
$$

Thus, $x_{o}$ and $y_{o}$ represent the sum of the actual and apparent translations in their respective directions and are used to correct the image.

\section{Signal Processing}

The ONAV signal processing was performed on a custom high-speed MR fluoroscopy image-reconstruction system (19), which included an array processor (MC-RACE, Mercury Computer Systems, Chelmsford, MA) and host computer (SPARC5, Sun Microsystems, Sunnyvale, CA) that were interfaced to the MRI scanner $(15,20)$. The detected rotation was measured by determining the shift, $\alpha$, which minimized the sum of the squared difference between the reference and current ONAV signals (21). For each ONAV, once the rotation was detected, a least-squares fitting function was applied to the phase difference from Eq. [2] between the current and reference ONAV signals to estimate the translation within the plane of the ONAV, as described in Grimm et al. (18).

\section{Implementation of Corrections}

After detection of the rotations and translations, the values of the three measured rotations and through-plane translation were encoded in a single variable for transfer to the MRI scanner. These values were then decoded by the MRI scanner, and correction for the rotations and throughplane translation was performed prospectively by appropriate adjustment of the gradient rotation matrix and $\mathrm{RF}$ excitation frequency prior to excitation of the subsequent imaging sequence.

Since application of orthogonal rotation matrices is noncommutative, and the angles of rotation were measured about the axes of the original gradient rotation matrix, the standard rotation matrices used to describe rotation of an object do not apply. Instead, the three orthogonal angles of rotation can be considered components of a single rotation of magnitude $\phi$ about the unit vector $\hat{n}$, where

$$
\phi=\sqrt{\alpha^{2}+\beta^{2}+\gamma^{2}}
$$

and

$$
\hat{n}=\frac{\alpha}{\phi} \hat{e}_{1}+\frac{\beta}{\phi} \hat{e}_{2}+\frac{\gamma}{\phi} \hat{e}_{3}
$$

$\alpha$ is the measured rotation about the unit vector $\hat{e}_{1}$ (sagittal rotation), $\beta$ is the measured rotation about the unit vector $\hat{e}_{2}$ (coronal rotation), $\gamma$ is the measured rotation about the unit vector $\hat{e}_{3}$ (axial rotation), and $\left(\hat{e}_{1}, \hat{e}_{2}, \hat{e}_{3}\right)$ are the perpendicular unit vectors describing the original frame of reference, or in other words, the gradient rotation matrix. If $\Re(\phi, \hat{n})$ is the rotation through angle $\phi$ about unit vector $\hat{n}$, the new frame of reference $\left(\hat{f}_{1}, \hat{f}_{2}, \hat{f}_{3}\right)$ can mathematically be described as (22)

$$
\begin{aligned}
\hat{f}_{i}=\Re(\phi, \hat{n}) \hat{e}_{i}= & \hat{e}_{i} \cos \phi+\left(\hat{n} \times \hat{e}_{i}\right) \sin \phi \\
& +\hat{n}\left(\hat{n} \cdot \hat{e}_{i}\right)(1-\cos \phi) \quad(i=1,2,3) .
\end{aligned}
$$

Note that this equation reduces to the standard rotation matrices given only one nonzero component of $\hat{n}$, and that for a rotation of zero, $\hat{n}$ is undefined. Thus, the gradient rotation matrix was updated to correct for rotation according to Eq. [4] only when rotation was detected.

Correction for in-plane translation was performed retrospectively by applying a phase modulation to the complex data set after row-flipping the even echoes relative to the odd echoes of the EPI data set and before EPI image reconstruction. Correction for in-plane translation in the readout direction could be performed prospectively by adjusting the center frequency of the receiver according to the polarity of each echo of the EPI readout. However, the additional $50 \mathrm{msec}$ necessary to transfer the in-plane translation information is an unacceptable trade-off when no 
advantage is gained from prospective correction of inplane translation, and near real-time correction can be made on the array processor.

After readout of the first ONAV signal ( $5.6 \mathrm{msec}$ ), the data is transferred from the receiver to the array processor (25 msec). During the data transfer, the second and third ONAV signals are acquired. As the second ONAV signal is being transferred ( $25 \mathrm{msec}$ ), calculation of the rotation and translation of the first ONAV takes place. Likewise, rotation and translation calculations for the second ONAV occur during transfer of the third ONAV signal (25 msec). Calculation of the rotation (3 $\mathrm{msec})$ and translation (3 msec) of the third ONAV takes place after the third ONAV signal has been transferred. Finally, transfer of rotation and through-plane translation values from the array processor to the MR scanner and update of the rotation matrix and slice offset of the subsequent EPI sequence requires approximately $70 \mathrm{msec}$. Thus, the total time requirement to comfortably include all of the steps from application of the first ONAV excitation to alteration of the EPI imaging parameters is $160 \mathrm{msec}$.

\section{Phantom}

To assess the accuracy of complex motion detection, a phantom capable of applying precisely known rotations and translations in multiple planes was necessary. Our previous studies had only considered single-plane rotational and translational movement (16). This setup was inadequate for the current work which focused on how the method would perform during combinations of rotations and translations. In addition, the geometry of the older phantom itself was not representative of the human head. Thus, a motion phantom was designed with computercontrolled stepper motors controlling sagittal rotation, axial rotation, and $z$ translation. The imaged part of the phantom was the shape of a human skull which was generated by a CT modeler and then filled with gel. The skull was modeled at $75 \%$ scale to reduce the moment of inertia and improve the accuracy of applied axial rotations. Glass rods were inserted into the skull phantom in a three dimensional axes formation to serve as fiducials, with the intersection of the rods located at the center of axial rotation.

Calibration studies of the phantom indicated that motions were performed with an accuracy of $\pm 0.13^{\circ}$ for sagittal rotations, $\pm 0.5^{\circ}$ for axial rotations, and $\pm 0.2 \mathrm{~mm}$ for translations. It should be noted that because of the geometry of the motion phantom, an applied sagittal rotation $(\alpha)$ moved the plane of the applied axial rotation $(\gamma)$ out of alignment with the axial imaging plane, such that the applied axial rotation had components in each of the axial and coronal planes, given by $\gamma \cos \alpha$ and $\gamma \sin \alpha$ respectively.

\section{Multiple ONAV Pulse Sequence}

Previously, we had used an ONAV excitation pulse that was $5 \mathrm{~mm}$ thick (16). This limited slice thickness posed a problem for subsequent ONAVs in that the object "seen" by the subsequent ONAV had a saturation band which essentially served as a stationary fiducial, independent of whether the object had moved. This corrupted the ONAV signal, making detection of motion by any ONAV other than the initial ONAV very poor. We subsequently determined that this problem could be avoided by using an ONAV with slice thickness greater than the imaged object (23).

For maximum efficiency, it is desirable to keep both the delay between ONAV acquisition and correction $\left(\tau_{\text {NAV }}\right)$ as well as the internav delay (NAV TR) as short as possible (Fig. 1). Hence, each ONAV pulse will produce some degree of spin saturation which will be present during subsequent readouts in the ONAV sequence. We experimentally identified a flip angle $\left(10^{\circ}\right)$ for gradient-recalled ONAVs which provided optimal SNR and sensitivity to motion in a series of closely spaced ONAVs (23).

\section{Experimental Aim 1}

\section{Multiaxis Correction of Complex Motions}

The first aim was to assess the sensitivity of the multiple ONAV method to various combinations of simultaneous rotation and translation. The three ONAVs were prescribed as gradient echo sequences with $\mathrm{TE}=3 \mathrm{msec}$, slice thickness $=240 \mathrm{~mm}$, flip angle $=10^{\circ}, 24-\mathrm{cm} \mathrm{FOV}$, and NAV TR $=11$ msec. The values of $k_{\rho}$ for the skull phantom were selected for greatest accuracy for each of the ONAV planes to be $14.65 / \mathrm{FOV}=0.61 \mathrm{~cm}^{-1}$ for the coronal and sagittal planes, and 17.35/FOV $=0.72 \mathrm{~cm}^{-1}$.

For each test, motions in all six degrees of freedom were measured and compared with the expected motions applied by the motion phantom using the Limits of Agreement (LOA) statistic, defined as the mean difference between the measured motion and the expected motion \pm 2 standard deviations of the difference (24). The expected motions for axial and sagittal rotations were the applied rotations in their respective planes. The expected motions for coronal rotations were equal to $\gamma \sin \alpha$, the component of axial rotation in the coronal plane resulting from the geometrical setup of the phantom. With knowledge of the center of rotation and applied rotations, the expected apparent translation could be calculated. Let $\mathbf{X}_{r}$ be the position of the center of rotation $\left(x_{r}, y_{r}, z_{r}\right)$ with respect to the center of the magnet $(0,0,0)$ in the frame of reference of the original gradient rotation matrix, and $\mathbf{X}_{r}^{\prime}$ be the position of the center of rotation in the frame of reference of the updated gradient rotation matrix. The apparent translation $\Delta \mathbf{X}_{\text {app }}$ is the difference between these two values

$$
\begin{aligned}
\Delta \mathbf{X}_{\mathrm{app}} & =\mathbf{X}_{r}^{\prime}-\mathbf{X}_{r}=\mathfrak{R}(-\phi, \hat{n}) \mathbf{X}_{r}-\mathbf{X}_{r} \\
& =\left[\hat{n}\left(\hat{n} \cdot \mathbf{X}_{r}\right)-\mathbf{X}_{r}\right](1-\cos \phi)-\left(\hat{n} \times \mathbf{X}_{r}\right) \sin \phi,
\end{aligned}
$$

and the expected translation was then the sum of the applied translation and the apparent translation.

In order to assess the consistency of motion detection, several permutations of multidirectional motion were tested. In test 1 , for each axial rotation of $0^{\circ}, \pm 3^{\circ}, \pm 5^{\circ}$, $\pm 10^{\circ}$, the phantom was simultaneously rotated in the sagittal direction at a finer resolution to $0^{\circ}, \pm 1^{\circ}, \pm 2^{\circ}, \pm 3^{\circ}$, $\pm 4^{\circ}, \pm 5^{\circ}, \pm 7^{\circ}$ and $\pm 10^{\circ}$. In test 2 , to analyze the generalizability of the results, the sampling resolutions were reversed from test 1 such that for each sagittal rotation of $0^{\circ}$, 
Table 1

Experimental Aims 1 and 2

\begin{tabular}{|c|c|c|c|c|c|c|}
\hline \multicolumn{7}{|c|}{ a. Precision of detected rotations (degrees) } \\
\hline \multirow{2}{*}{$\begin{array}{l}\text { No. of } \\
\text { ONAV } \\
\text { sets }\end{array}$} & \multicolumn{2}{|c|}{ Coronal rotation } & \multicolumn{2}{|c|}{ Sagittal rotation } & \multicolumn{2}{|c|}{ Axial rotation } \\
\hline & $\begin{array}{l}\text { Small motions } \\
\quad(n=72)\end{array}$ & $\begin{array}{l}\text { Large motions } \\
\qquad(n=128)\end{array}$ & $\begin{array}{l}\text { Small motions } \\
\quad(n=72)\end{array}$ & $\begin{array}{l}\text { Large motions } \\
\qquad(n=128)\end{array}$ & $\begin{array}{l}\text { Small motions } \\
\quad(n=72)\end{array}$ & $\begin{array}{l}\text { Large motions } \\
\qquad(n=128)\end{array}$ \\
\hline 1 & -0.72 to 0.74 & -1.52 to 1.80 & -0.31 to 0.31 & -0.43 to 0.41 & -0.47 to 0.48 & -0.64 to 0.54 \\
\hline 2 & -0.33 to 0.33 & -0.58 to 0.53 & -0.28 to 0.30 & -0.49 to 0.48 & -0.43 to 0.46 & -0.72 to 0.55 \\
\hline \multicolumn{7}{|c|}{ b. Precision of detected translations $(\mathrm{mm})$} \\
\hline No. of & \multicolumn{2}{|c|}{$x$-Translation } & \multicolumn{2}{|c|}{$y$-Translation } & \multicolumn{2}{|c|}{$z$-Translation } \\
\hline $\begin{array}{l}\text { ONAV } \\
\text { sets }\end{array}$ & $\begin{array}{l}\text { Small motions } \\
\quad(n=55)\end{array}$ & $\begin{array}{l}\text { Large motions } \\
\quad(n=96)\end{array}$ & $\begin{array}{l}\text { Small motions } \\
\quad(n=55)\end{array}$ & $\begin{array}{l}\text { Large motions } \\
\quad(n=96)\end{array}$ & $\begin{array}{l}\text { Small motions } \\
\quad(n=55)\end{array}$ & $\begin{array}{l}\text { Large motions } \\
\qquad(n=96)\end{array}$ \\
\hline 1 & $\begin{array}{c}-0.23 \text { to } 0.28 \\
*\end{array}$ & $\begin{array}{c}-0.39 \text { to } 0.54 \\
*\end{array}$ & $\begin{array}{c}-0.48 \text { to } 0.48 \\
*\end{array}$ & $\begin{array}{c}-0.93 \text { to } 0.91 \\
*\end{array}$ & $\begin{array}{c}-0.27 \text { to } 0.33 \\
*\end{array}$ & $\begin{array}{c}-1.41 \text { to } 1.45 \\
*\end{array}$ \\
\hline 2 & -0.17 to 0.17 & -0.31 to 0.28 & -0.32 to 0.30 & -0.67 to 0.57 & -0.14 to 0.22 & -0.53 to 0.58 \\
\hline
\end{tabular}

The angles of Table 1a show the limits of agreement (mean difference \pm 2SD) between the applied rotations and the detected rotations as measures of the precision of rotational detection. The distances of Table $1 \mathrm{~b}$ show the limits of agreement between the applied translations and the detected translations as measures of the precision of translational detection. Motions in which the magnitude of rotation in all three orthogonal planes was $\leq 4^{\circ}$ and with through-plane translation of magnitude $\leq 4 \mathrm{~mm}$ were defined as small motions. Motions consisting of rotations of magnitude $>4^{\circ}$ and $\leq 8^{\circ}$ in any of the three orthogonal planes, or through-plane translation of magnitude $>4$ mm and $\leq 8 \mathrm{~mm}$, were defined as large motions.

*Statistically significant change in accuracy of detection between ONAV set 1 and ONAV set 2 ( $P<0.001$, paired $t$ test).

$\pm 3^{\circ}, \pm 6^{\circ}$, and $\pm 10^{\circ}$, the phantom was rotated in the axial direction from $-10^{\circ}$ to $+10^{\circ}$ at a finer resolution of $2^{\circ}$ increments. In test 3 , for each axial rotation of $0^{\circ}, \pm 3^{\circ}, \pm 6^{\circ}$, and $\pm 10^{\circ}$, the phantom was translated in the $z$ direction from $-10 \mathrm{~mm}$ to $+10 \mathrm{~mm}$ in 2 -mm increments. In test 4 , for each sagittal rotation of $0^{\circ}, \pm 3^{\circ}, \pm 6^{\circ}$, and $\pm 10^{\circ}$, the phantom was translated in the $z$ direction from $-10 \mathrm{~mm}$ to $+10 \mathrm{~mm}$ in 2-mm increments.

\section{Experimental Aim 2}

\section{Multiaxis Motion Correction With Two Sets of ONAVS}

The second aim was to test the hypothesis that after the measurement of rotations by the three orthogonal ONAVs, rotating the gradients to correct for the measured rotations and acquiring a second set of three orthogonal ONAVs would give more accurate final measurements of rotation and translation. The tests of experimental aim 1 were repeated with the following modification: immediately following the update of the gradient rotation matrix dictated by the first set of ONAVs, a second set of three orthogonal ONAVs was acquired. Both the first and second sets of ONAV signals acquired before the initial baseline multislice image cycle in the time series were saved as reference waveforms. The signals from both ONAV sets 1 and 2, acquired before any subsequent multislice image cycles, were then compared to their respective reference waveforms. Any detected rotation from the second set of ONAVs was added to the detected rotation of the first set, since the rotation detected from the first set had already been corrected. Detected translation from the second set of ONAVs was the absolute translation, since no correction of translation took place between ONAV sets 1 and 2. The sum of the detected rotations from both sets of ONAVs and the through-plane translation measured by the second set of ONAVs were then sent to the MRI scanner to update the EPI acquisition. Because the original ONAV sequence (occupying time $\tau_{\text {NAV }}$ of Fig. 1) was essentially duplicated, this modification required an additional $160 \mathrm{msec}$, for a total of $320 \mathrm{msec}$ of acquisition and processing for both ONAV sets.

\section{Experimental Aim 3}

\section{In Vivo Anatomic Images}

The third aim was to demonstrate that the accuracy of rotational and translation correction in all six degrees of freedom that was demonstrated in the motion phantom could be achieved in vivo. To test this hypothesis, a volunteer was instructed to deliberately perform head rotations with components of rotation in multiple planes at prescribed points in the imaging sequence. The $k$-space radii for the ONAV acquisitions were determined empirically and set at $13 / \mathrm{FOV}=0.54 \mathrm{~cm}^{-1}$ for the coronal and sagittal planes, and $9 / \mathrm{FOV}=0.38 \mathrm{~cm}^{-1}$ for the axial plane. The multislice EPI scan parameters were TR $=4000 \mathrm{msec}$ and $\mathrm{TE}=45 \mathrm{msec}$, with a $64 \times 64$ acquisition matrix. In the multislice EPI cycle, two axial anatomical imaging planes were prescribed, the first of which remained uncorrected, and the second of which was corrected for rotational and translational motion. The slices were prescribed such that they intersected the lateral ventricles and had a large enough interslice gap that the uncorrected image did not overlap with the corrected image. Thus, corrected and uncorrected image locations could be compared following the same physical motion.

\section{Effect of ONAVs on fMRI Activation}

In order to demonstrate that spin saturation effects due to the ONAV acquisition would not interfere with the detec- 

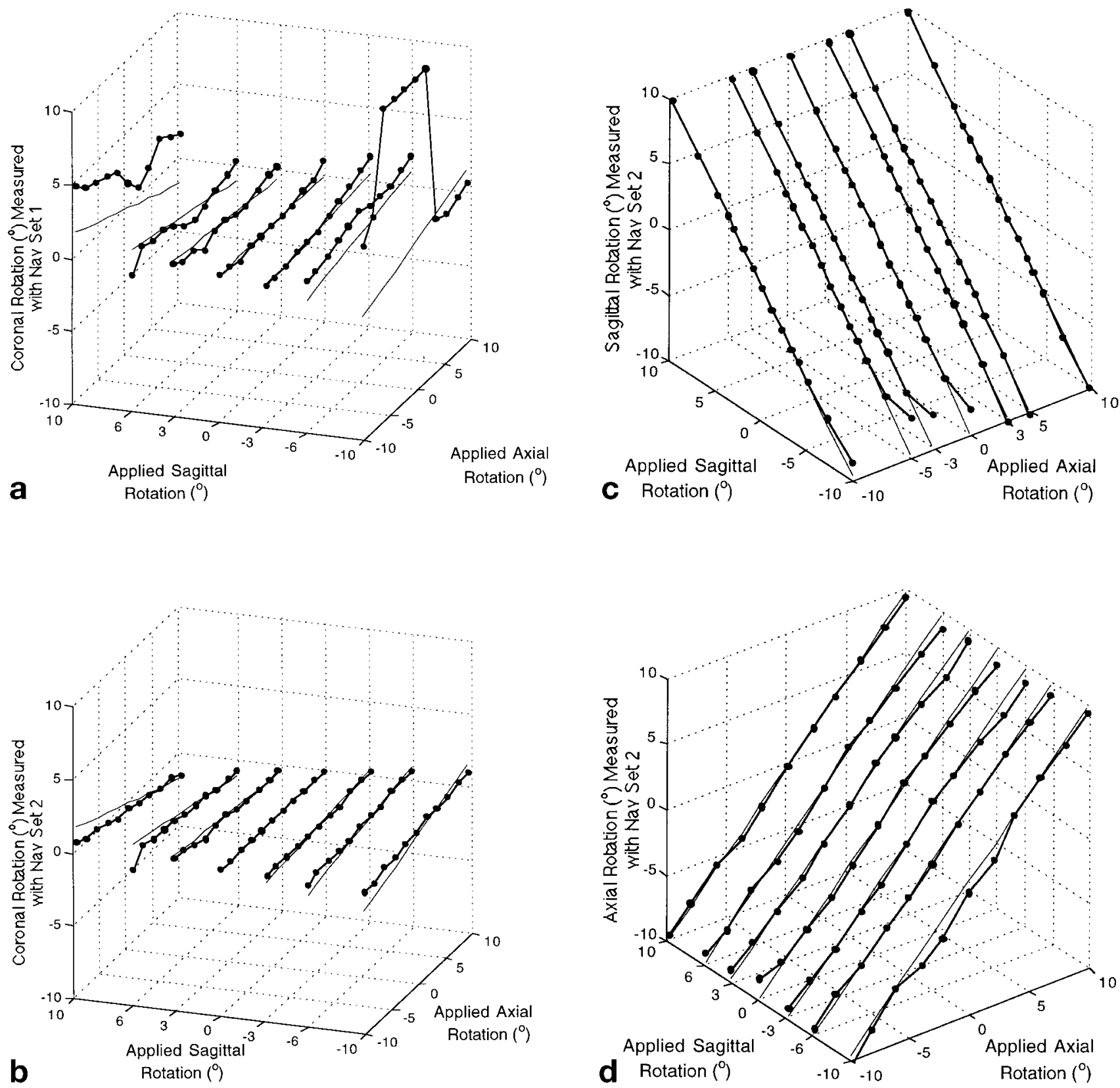

FIG. 2. Detection of rotation. The detected and implemented rotations (dots) are plotted as functions of the sagittal and axial rotations applied to the motion phantom, and compared with the expected rotations (hairlines). The difference between the plots of coronal rotation measured with ONAV set 1 (a) and coronal rotation measured with ONAV set 2 (b) demonstrate improved detection of rotation after updating the gradient rotation matrix with the measurements from ONAV set 1. In order to show actual data for motions detected by all three planar ONAVs, the detected and implemented rotations in the sagittal plane (c) and the axial plane (d) are shown, but only for ONAV set 2.

tion of blood oxygenation level-dependent (BOLD) signal changes, multislice fMR imaging was performed both with and without the ONAV sequence in a single volunteer. The ssEPI scanning parameters were $\tau_{\mathrm{NAV}}=320 \mathrm{msec}$ for two sets of ONAVs, TR $=1700 \mathrm{msec}$, TE $=45 \mathrm{msec}$, flip angle $=72^{\circ}$, FOV $24 \mathrm{~cm}$ with a $64 \times 64$ imaging matrix, and nine 5 -mm-thick axial slices prescribed through the anatomic pre- and postcentral gyri. The stimulus paradigm consisted of an alternating hand, sponge-squeezing task with 6 half cycles and 12 images per half cycle. The fMRI time series were analyzed in AFNI (9), using a cross- correlation analysis with a sinusoidal function at the primary stimulus frequency.

\section{Real-Time Motion-Corrected fMRI}

In order to demonstrate improved detection of activation using the real-time prospective correction method, fMR images were acquired while a volunteer deliberately moved his head at prescribed points in the time series. The ssEPI scanning parameters were the same as above, except with $\tau_{\mathrm{NAV}}=520 \mathrm{msec}$ to ensure that all detected rotations 


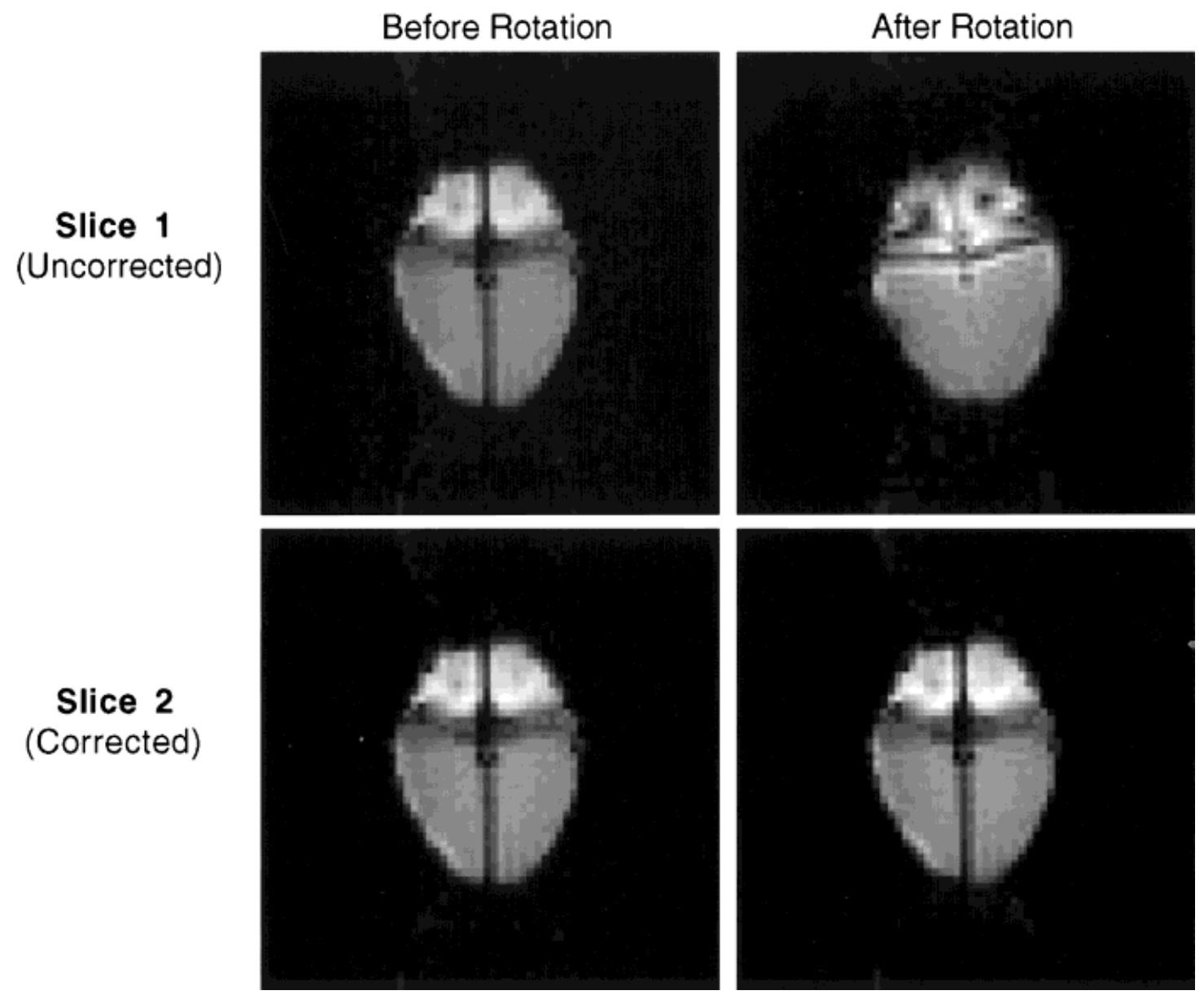

FIG. 3. Correction of motion: phantom images. Two ordered image pairs (uncorrected, corrected) were acquired to demonstrate real-time correction of complex motion with the multiaxis motion phantom. The slices were acquired sequentially at the same location through the glass rods in a given TR, undergoing identical physical motions. The applied motions were $6^{\circ}$ of axial rotation, $2^{\circ}$ of sagittal rotation, and $6 \mathrm{~mm}$ of through-plane translation. The position of the uncorrected slice (top row) changes after motion, with in-plane rotation visible from the angle of the horizontal glass rod, and through-plane rotation and translation visible from the shortening of the vertical glass rod and cut through the orbits. The position of the corrected slice (bottom row) remains the same before and after motion.

were received by the scanner for update before the next images. One fMRI acquisition had correction disabled, but the ONAVs were still executed to record the magnitude of motion the volunteer performed. A second fMRI acquisition had correction enabled. A cross-correlation analysis thresholded at $r=0.6$ was applied to both time series. The image set immediately preceding a change in detected motion of greater than $1^{\circ}$ in any plane was discarded prior to cross-correlation.

\section{RESULTS}

Experimental Aim 1

\section{Multiaxis Correction of Complex Motions}

The data from the tests for experimental aim 1 are summarized in the first row of Tables 1a and 1b. The multiaxis ONAV method had subdegree and submillimeter accuracy for combinations of motions defined as "small:" rotations less than $\pm 4^{\circ}$ and translations less than $\pm 4 \mathrm{~mm}$. For "large" motions up to $\pm 8^{\circ}$ and $\pm 8 \mathrm{~mm}$, subdegree accuracy was still observed for the detection of sagittal and axial rotations, and submillimeter accuracy was observed for the detection of $x$ and $y$ translations. However, detection of $z$ translation and coronal rotation were at times inaccurate by more than $1 \mathrm{~mm}$ or $1.5^{\circ}$ respectively.

\section{Experimental Aim 2}

\section{Multiaxis Motion Correction With Two Sets of ONAVs}

The data from the tests for experimental aim 2 are summarized in the second row of Tables 1a and 1b. Using two sets of ONAVs, the multiaxis ONAV method had subdegree and submillimeter accuracy for combinations of motions that included rotations less than $\pm 8^{\circ}$ and translations less than $\pm 8 \mathrm{~mm}$. Detection of small motions was still more accurate than detection of large motions. Improvements in the detection of coronal rotation and $x, y$, and $z$ translations from the first ONAV set to the second ONAV set were statistically significant with $P<0.001$ (paired $t$ test). Detection of sagittal or axial rotation was not significantly different between the first and second sets of ONAVs, with $P>0.05$. 


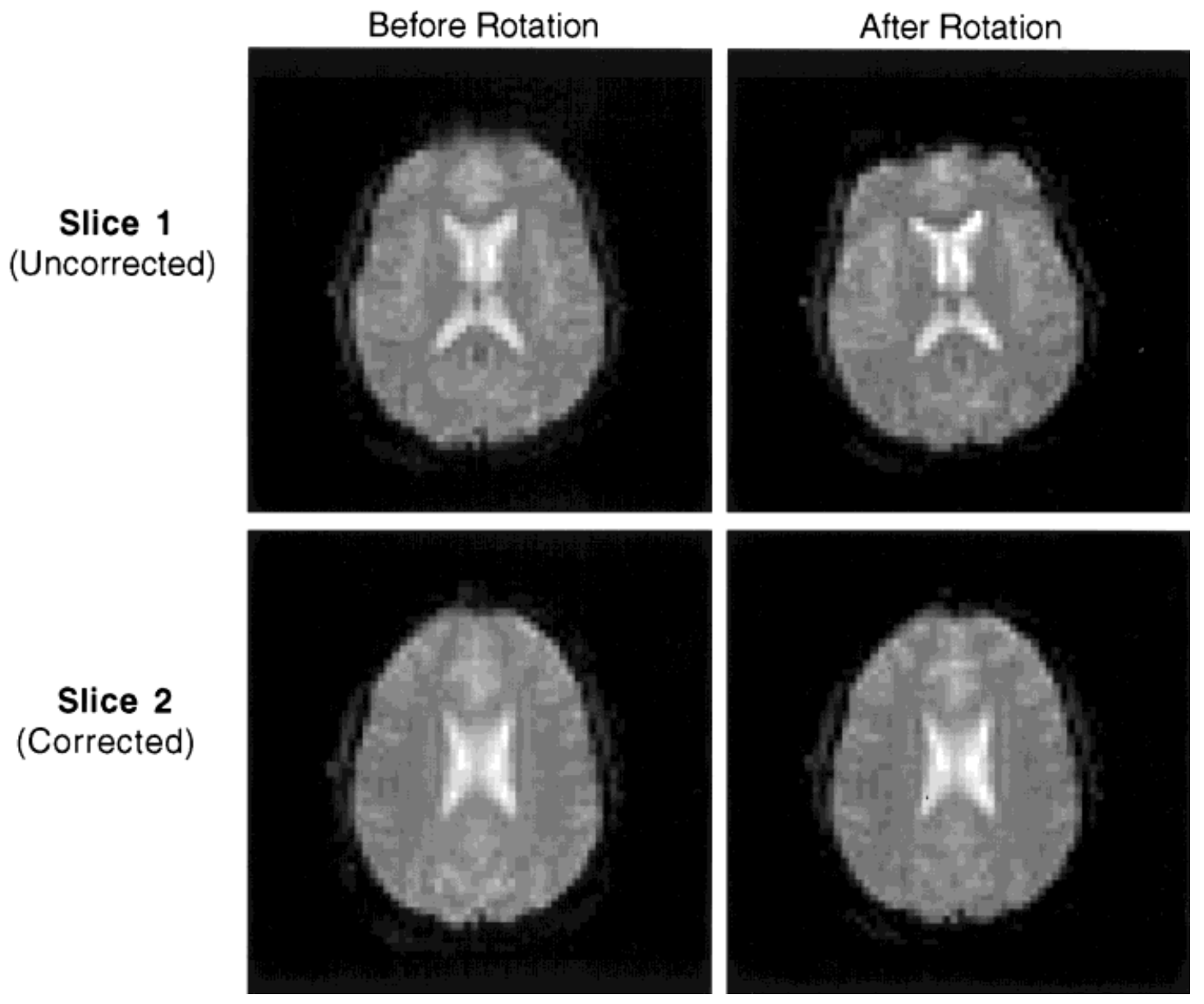

FIG. 4. Correction of motion: in vivo anatomic images. Two ordered image pairs (uncorrected, corrected) were acquired to demonstrate real-time correction of complex motion in a volunteer subject. The slices were acquired sequentially at different anatomic locations in a given TR, undergoing identical physical rotations. The appearance of the uncorrected slice (top row) changes after rotation, with in-plane rotation visible from the angle of the midline with respect to the image, and through-plane rotation apparent from the change in signal dropout near the frontal sinuses and the thinner profile through the midpoint of the ventricles after rotation. The appearance of the corrected slice (bottom row) remains the same before and after rotation. The measured rotations were $4^{\circ}$ in the axial plane and $6^{\circ}$ in the sagittal plane.

Figure 2 shows examples of the detected rotations for tests 1 and 2. Figure 3 displays the EPI images of the head phantom for one combination of complex motions, demonstrating accurate reorientation of the glass rod fiducials within the imaging slice.

\section{Experimental Aim 3}

\section{In Vivo Anatomic Images}

Figure 4 shows the in vivo results for a complex rotation. Correction of in-plane rotation is clearly apparent. Through-plane rotation is not as easily visualized in the uncorrected image, but differences in the morphology of the ventricular system and signal dropout near the frontal sinuses are apparent when comparing the pre- and postrotation image pair without correction. Both the throughplane and in-plane rotations have been eliminated in the corrected image pair.

\section{Effect of ONAVs on fMRI Activation}

Figure 5 shows BOLD activation maps acquired both with and without the ONAV sequence. The similarity in the maps demonstrates that saturation effects from the ONAV acquisition do not interfere with the subsequent detection of BOLD signal.

\section{Real-Time Motion-Corrected fMRI}

Figure 6 shows BOLD activation maps acquired with uncorrected and corrected fMRI sequences during deliberate, stimulus-correlated motion. In the uncorrected image, false-positive activation in areas other than the anatomical motor cortex is pronounced. This, along with loss of activation inside the motor cortex, makes determination of the true activated pixels difficult. In the motion corrected image, the true activation along the central sulcus is more prominent and the amount of false-positive activation detected in areas other than the sensorimotor cortex is markedly reduced. As a result, motor activation is much more clearly visualized.

\section{DISCUSSION}

Interimage global head motion is problematic in all fMRI studies, particularly in clinical implementations. Retro- 


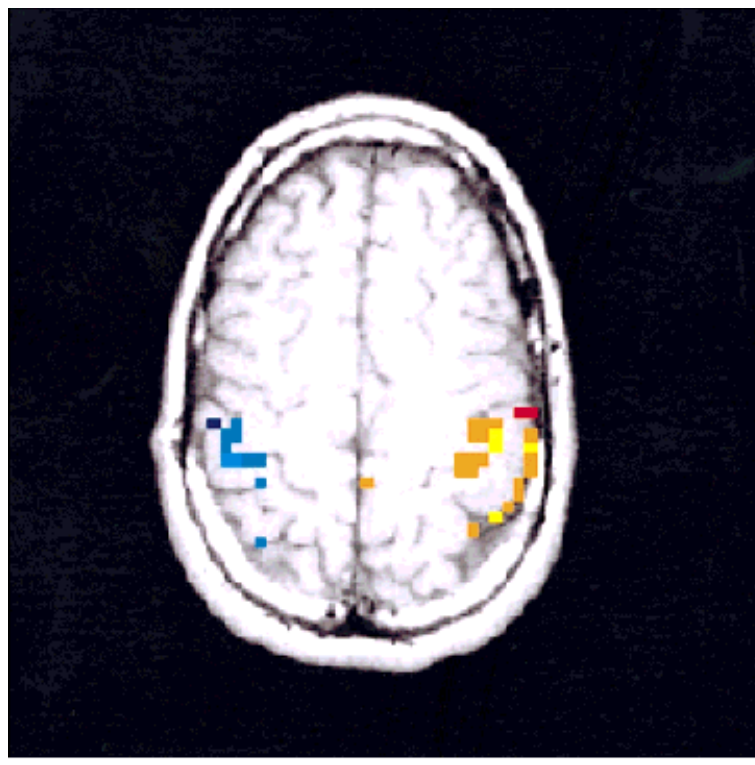

Acquired without ONAVs

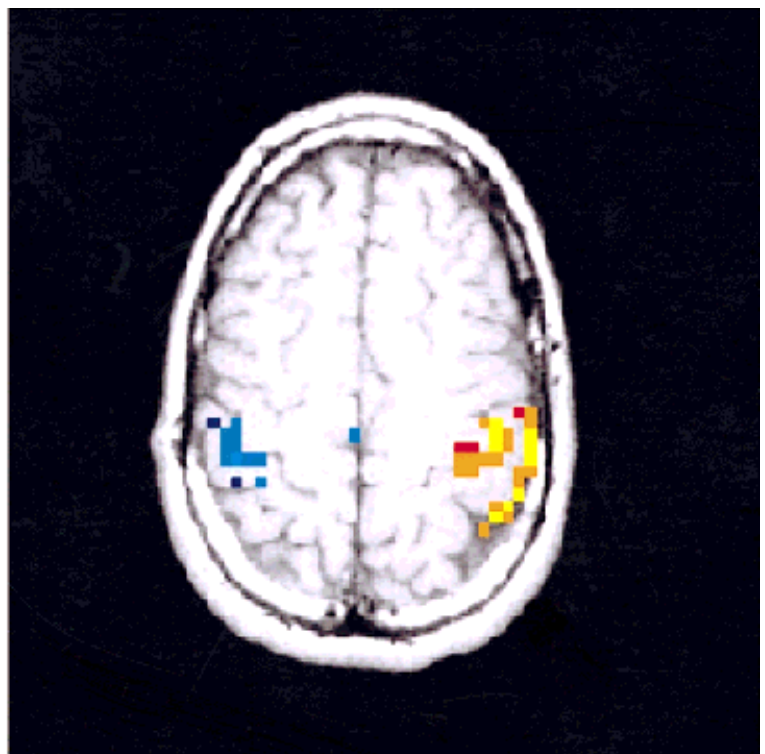

Acquired with ONAVs

FIG. 5. Effect of ONAVs on sensitivity to the BOLD phenomenon. Two functional MR imaging runs were acquired during a sensorimotor stimulation task: one without ONAVs (left), and one with ONAVs (right). The similar appearance of the functional maps demonstrates that incorporation of ONAVs into the EPI sequence does not interfere with detection of BOLD contrast.

spective motion correction algorithms can improve fMRI image results, but have drawbacks including blurring due to interpolation and image transformations, poor registration due to local intensity changes from the BOLD signal (12), and the potential to introduce false-positive (25) or mask true-positive activation. Through-plane motion presents more serious challenges for retrospective motion correction algorithms, such as aliasing resulting from interpolation in the slice-select direction, disruption of the spin history, and loss of physiologic information due to incorrect combination of data from adjacent slices that may have been acquired several seconds apart in the time series. Pulse sequence modifications to improve the accuracy of interpolation in the slice-select direction may reduce the precision of the slice selection profiles, require overlapping slices, and make the assumption that spin history is spatially independent which may not be true in the presence of long $T_{1}$ tissues $(26,27)$. Three-dimensional functional image acquisitions would allow for retrospective correction of through-plane motion. However, 3D acquisitions cannot be acquired in a single shot without excessively coarse spatial resolution, and multishot 3D acquisitions introduce the possibility of intraimage artifacts from head movement between shots.

\section{Experimental Aims 1 and 2}

Multiaxis Correction of Complex Motions and Multiaxis Motion Correction With Two Sets of ONAVS

The results of experimental aim 2 showed an improvement in the accuracy of the second set of ONAVs over the first set of ONAVs. The reason for this improvement is that each ONAV, like the imaging acquisition it is attempting to correct, is susceptible to through-plane motion problems itself. With the volume excitation, each ONAV samples the $k$-space of the projection of the imaged object through a given plane. Any rotation orthogonal to the plane of that ONAV changes the projection in that plane, thus altering the ONAV signal. The effect of rotation orthogonal to a given ONAV is that the resulting alteration in the ONAV signal has the potential to degrade the fidelity of the least squares fit both for the determination of rotation and translation. Figure $7 \mathrm{a}$ and $\mathrm{b}$ demonstrates how the coronal ONAV signal changes in the presence of sagittal rotation. The ONAV signal is not affected by through-plane translation because, as long as the object remains in the excitation volume, the projection does not change.

The second set of ONAVs improved rotational and translational accuracy because the gradient rotation matrix is corrected by the results of the first set of ONAVs. Thus, the projections sampled by each ONAV in the second set better match the projections sampled by the respective reference ONAV, and more accurate detection of rotation and translation can be achieved. Figure 7c shows how updating the rotation matrix to account for sagittal rotation improves the agreement between the coronal ONAV signal and its reference ONAV signal.

The second set of ONAVs also improves measurement of translation because apparent translation in both planes of detection are measured. In each set of ONAVs, translation in each physical direction is measured by two separate ONAVs. For example, $z$ translation is measured by both the coronal and sagittal ONAVs. The average of these values is sent to the MRI scanner for correction of throughplane translation. To continue with this example, if a sagittal rotation occurs, when analyzing the phase of the sagittal ONAV signal, the apparent translation in both the 

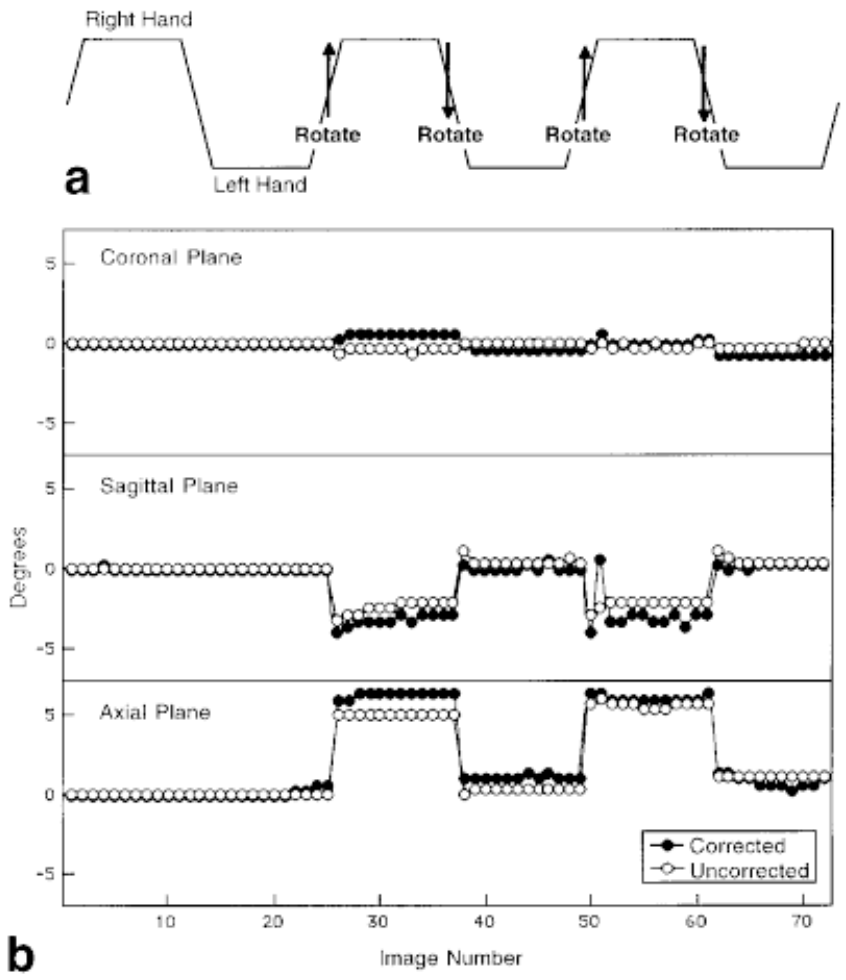

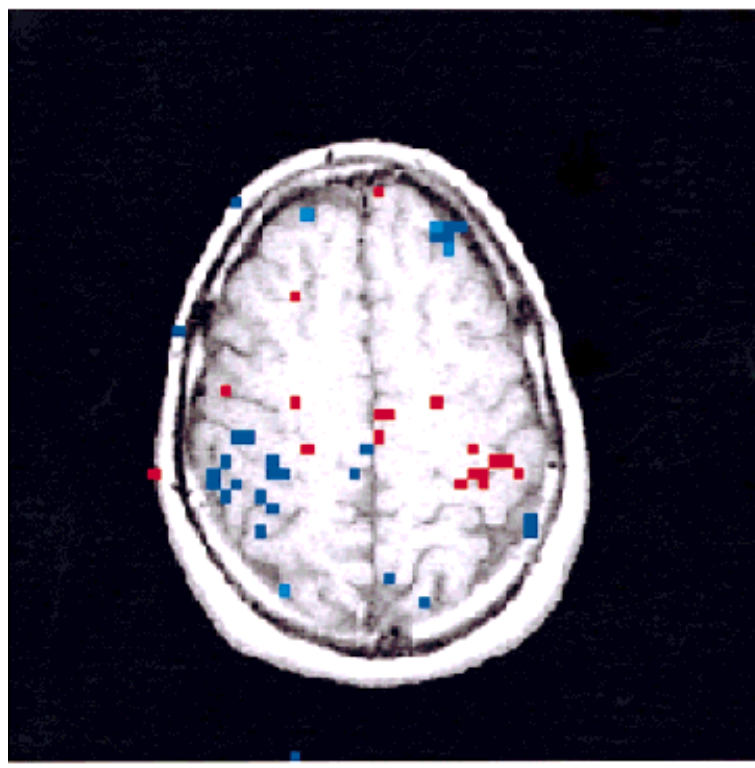

C
Uncorrected

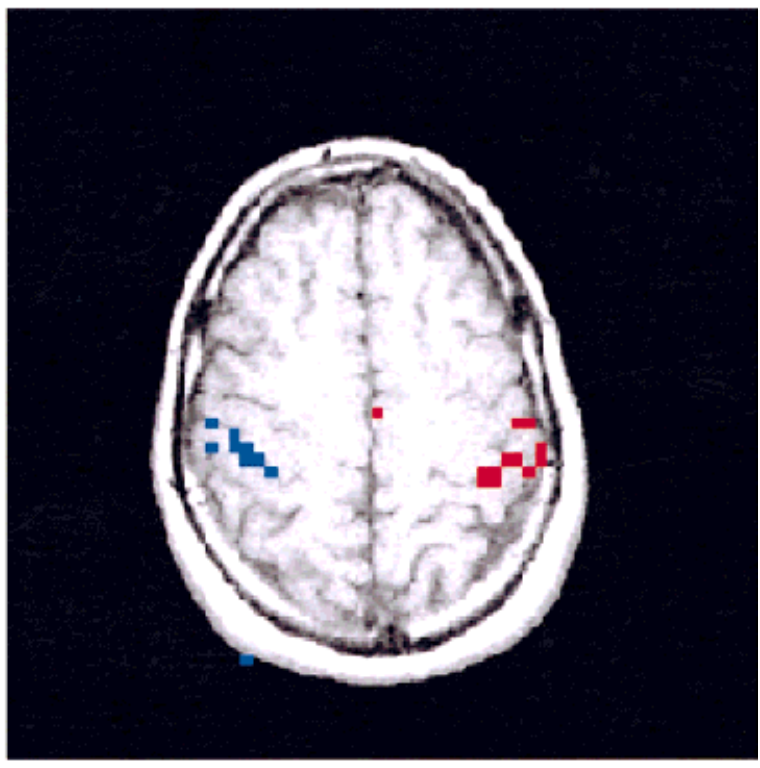

Corrected

FIG. 6. Motion-corrected fMRI. Head rotation was incorporated into an alternating hand, sponge-squeezing activation paradigm (a). Stimulus correlated intentional head rotation occurred four times within the fMRI acquisitions. Two functional MR imaging runs were acquired: one with ONAVs but with correction disabled, and one with correction enabled. Motion was recorded by the ONAVs (b), and was found to be about equal for both the motion-corrected (dots) and motion-uncorrected studies (open circles). In the functional activation maps (c), motor activation is much more clearly visualized in the corrected image (right) than in the uncorrected image (left).

$y$ and $z$ directions is measured by shifting the phase of the reference waveform by the amount of measured rotation, $\Psi(\theta-\alpha)$ from Eq. [2]. This emulates the upcoming change in rotational frame of reference. There is no analogous way to account for the upcoming change in rotational frame of reference for the coronal ONAV because the sagittal rota- tion is a through-plane motion, and thus the coronal ONAV in the first set is incapable of producing an accurate measure of apparent $z$ translation. This results in discrepancies between the $z$ translation measured by the sagittal and coronal ONAVs. By correcting the gradient rotation matrix after the first set of ONAVs, the rotational frame of 

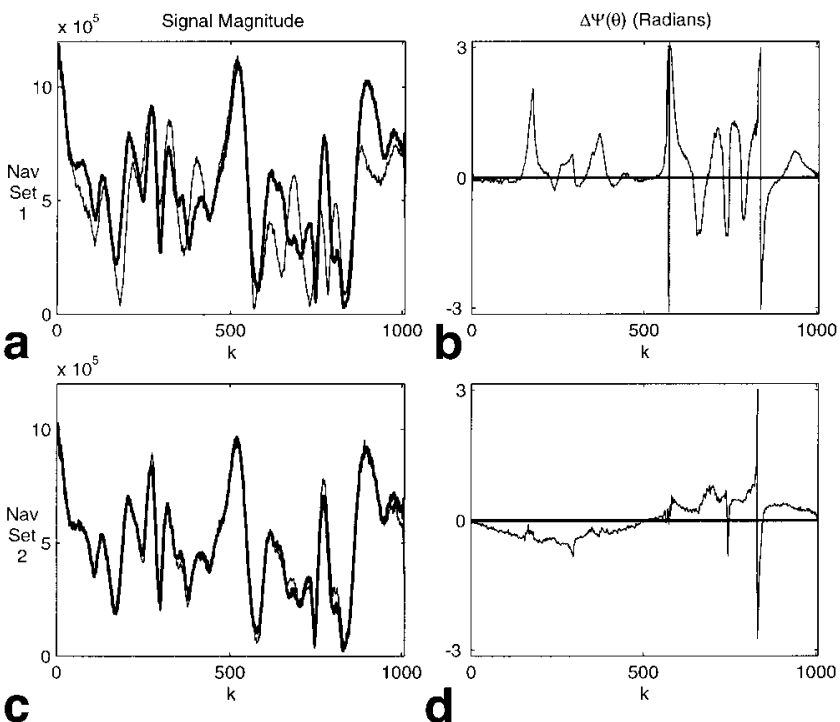

FIG. 7. Effects of through-plane rotation on ONAV signal. Rotation orthogonal to the ONAV plane of detection alters the subsequent ONAV signal (hairline) from the baseline ONAV signal (bold), degrading the fidelity of the least-squares fit (a) and analysis of the phase difference (b). Updating the gradient rotation matrix before acquisition of a second set of ONAVs corrects for the through-plane rotation, improving the fidelity of the least squares fit (c) and allowing for the detection of apparent translation from the phase difference (d). The above ONAV signals were acquired in the coronal plane before and after a $6^{\circ}$ sagittal rotation.

reference has already been corrected when the second ONAV set is acquired, and thus both the sagittal and coronal ONAV signals contain information about the apparent $z$ translation. This example can be generalized to all directions of translation. Figure $7 \mathrm{~d}$ shows the phase difference of the coronal ONAV after updating the gradient rotation matrix, from which the apparent translation information can then be gleaned.

Although using two sets rather than one set of ONAVs demonstrates an increase in accuracy, using more than two sets of ONAVs is impractical at this time because of the time overhead of the current implementation. When engineering advances, particularly reducing the time required for data transmission from the array processor to the scanner, make additional sets of ONAVs practical, any additional benefit of supplementary sets of ONAVs will have to be weighed against the processing time required.

Incorporating the cumulative record of detected rotation throughout the time series has an additional benefit in that distortion of the ONAV signal due to changes in the projection the ONAV samples is removed continuously, increasing the sensitivity of the ONAVs to future rotations. Also, updating the gradient rotation matrix for the ONAV acquisitions associated with each successive ONAV-image set means that correction of a given rotation occurs only once throughout the time series. Rotation between successive TRs is likely to be smaller, and thus more accurately detected, than rotations which accumulate over the course of an entire fMRI scan.

\section{Experimental Aim 3}

Effect of ONAVs on fMRI Activation

The decision to use a low flip-angle gradient echo ONAV with a volume excitation was made to decrease local saturation effects on any subsequent ONAVs. However, this approach also minimizes spin saturation during the EPI acquisition. Measurements made in a phantom show that spin saturation from the ONAV sequence (with two sets of ONAVs) only reduces the SNR of the EPI images by about $4 \%$. This small amount of spin saturation does not seem to have an appreciable negative impact on the sensitivity of the subsequent EPI images to the BOLD phenomenon (Fig. 5).

The gradient echo ONAV with whole volume excitation eliminates the stationary saturation band which would be induced in the following EPI images by a slice selective ONAV, removing the potential for masking areas of activation in fMRI images by saturating the tissue with the ONAV slice excitation (16). Hence, it is unnecessary to place the ONAVs in any specific position.

The ONAV FOV did include signal from the neck, which does not move with the head as a rigid body. To date, we have not recognized signal distortion originating from neck signal. However, future implementations will include a spatial saturation pulse to isolate ONAV signal of the head from that of the neck, perhaps providing better in vivo accuracy.

\section{CONCLUSION}

In this work we demonstrate the accuracy of a real-time interimage motion correction technique in the presence of complex global head motions. For a time penalty of only 320 msec per 1-4-sec TR, depending on the anatomical coverage desired, corrections of submillimeter and subdegree accuracy can be implemented prospectively. The loss of physiologic information from through-plane motion is prevented because the planes of acquisition are locked to the spatial frame of reference of the moving head. Although early, the results in volunteers demonstrate that real-time in vivo correction of multidirectional global head motion is both technically feasible and effective in improving the detection of task activation by fMRI.

\section{ACKNOWLEDGMENTS}

The authors thank Jillyn Freeman, Thomas Hulshizer, and Phillip Rossman.

\section{REFERENCES}

1. Hajnal JV, Myers R, Oatridge A, Schwieso JE, Young IR, Bydder GM. Artifacts due to stimulus correlated motion in functional imaging of the brain. Magn Reson Med 1994;31:283-291.

2. Jack CR, Lee CC, Riederer SJ. Functional brain imaging with a standard 1.5-T magnetic resonance imaging system. Acad Radiol 1995;2:916-923.

3. Friston KJ, Williams S, Howard R, Frackowiak RSJ, Turner R. Movementrelated effects in fMRI time-series. Magn Reson Med 1996;35:346-355.

4. Atlas SW, Howard RS, Maldjian J, Alsop D, Detre JA, Listerud J, D’Esposito M, Judy KD, Zager E, Stecker M. Functional magnetic resonance imaging of regional brain activity in patients with intracerebral gliomas: findings and implications for clinical management. Neurosurg 1996;38:329-338. 
5. Lee CC, Jack CR, Rossman PJ, Riederer SJ. Real-time reconstruction and high-speed processing in functional MR imaging. Am J Neuroradiol 1998;19:1297-1300.

6. Fitzsimmons JR, Scott JD, Peterson DM, Wolverton BL, Webster CS, Lang PJ. Integrated RF coil with stabilization for fMRI human cortex. Magn Reson Med 1997;38:15-18.

7. Woods RP, Mazziotta JC, Cherry SR. MRI-PET registration with automated algorithm. J Comput Assist Tomogr 1993;17:536-546.

8. Eddy WF, Fitzgerald M, Noll DC. Improved image registration by using Fourier interpolation. Magn Reson Med 1996;36:923-931.

9. Cox RW. AFNI: software for analysis and visualization of functional magnetic resonance neuroimages. Comput Biomed Res 1996;29:162173.

10. Strupp JP. Stimulate: a GUI based fMRI analysis software package. NeuroImage 1996;3:S607.

11. Maas LC, Frederick BD, Renshaw PF. Decoupled automated rotational and translational registration for functional MRI time series data: the DART registration algorithm. Magn Reson Med 1997;37:131-139.

12. Biswal BB, Hyde JS. Contour-based registration technique to differentiate between task-activated and head motion-induced signal variations in fMRI. Magn Reson Med 1997;38:470-476.

13. Kim B, Boes JL, Bland PH, Chenevert TL, Meyer CR. Motion correction in fMRI via registration of individual slices into an anatomical volume. Magn Reson Med 1999;41:964-972.

14. Wu DH, Duerk JL, Lewin JS, Friedman L. Correction for statistical biasing of interpolated fMR data after image registration schemes. In: Proceedings of the 4th Scientific Meeting of ISMRM, New York, 1996. p 344 .

15. Lee CC, Jack CR, Grimm RC, Rossman PJ, Felmlee JP, Ehman RL, Riederer SJ. Real-time adaptive motion correction in functional MRI. Magn Reson Med 1996;36:436-444.

16. Lee CC, Grimm RC, Manduca A, Felmlee JP, Ehman RL, Riederer SJ, Jack CR. A prospective approach to correct for interimage head rotation in fMRI. Magn Reson Med 1998;39:234-243.
17. Fu ZW, Wang Y, Grimm RC, Rossman PJ, Felmlee JP, Riederer SJ, Ehman RL. Orbital navigator echoes for motion measurements in magnetic resonance imaging. Magn Reson Med 1995;34:746-753.

18. Grimm RC, Riederer SJ, Ehman RL. Real-time rotation correction using orbital navigator echoes. In: Proceedings of the 5th Scientific Meeting of ISMRM, Vancouver, 1997. p 1899.

19. Wright RC, Riederer SJ, Farzaneh F, Rossman PJ, Liu Y. Real-time MR fluoroscopic data acquisition and image reconstruction. Magn Reson Med 1989;12:407-415.

20. Debbins JP, Riederer SJ, Rossman PJ, Grimm RC, Felmlee JP, Breen JF, Ehman RL. Cardiac magnetic resonance fluoroscopy. Magn Reson Med 1996;36:588-595.

21. Wang Y, Grimm RC, Felmlee JP, Riederer SJ, Ehman RL. Algorithms for extracting motion information from navigator echoes. Magn Reson Med 1996;36:117-123.

22. van Dam H, Louck JD, Biedenharn LC. Rotation and angular momentum. In: Lerner RG, Trigg GL, editors. Encyclopedia of physics. New York: VCH Publishers; 1991. p 1076-1081.

23. Ward HA, Lee CC, Grimm RC, Rossman PJ, Felmlee JP, Ehman RL, Riederer SJ, Jack CR. Multi axis real-time prospective motion correction in fMRI. In: Proceedings of the 6th Scientific Meeting of ISMRM, Sydney, 1998. p 296.

24. Bland JM, Altman DG. Statistical methods for assessing agreement between two methods of clinical measurement. Lancet 1986;i:307-310.

25. Wu DH, Lewin JS, Duerk JL. Inadequacy of motion correction algorithms in functional MRI: role of susceptibility induced artifacts. J Magn Reson Imaging 1997;7:365-370.

26. Noll DC, Boada FE, Eddy WF. A spectral approach to analyzing slice selection in planar imaging: optimization for through-plane interpolation. Magn Reson Med 1997;38:151-160.

27. Hajnal JV, Oatridge A, Murdoch J, Saeed N, Bydder GM. Multislice data acquisitions for multiplanar reformatting and image registration. In: Proceedings of the 6th Scientific Meeting of ISMRM, Sydney, 1998. p 560. 